\title{
Implementación de un control multiresolución empleando un dispositivo háptico
}

\author{
E. Mendoza-Monjaraz, J. A. Cruz-Tolentino, A. Jarillo-Silva, J. \\ Pacheco-Mendoza \\ Universidad de la Sierra Sur, \\ Miahuatlán de Porfirio Díaz, Oaxaca, México \\ \{eufra_mndoza\}@hotmail.com, \{jacruz, ajarillo, jpacheco\}@unsis.edu.mx \\ http://www.unsis.edu.mx
}

\begin{abstract}
Resumen. En este trabajo de investigación se realiza la implementación de un control multiresolución sobre un dispositivo háptico Novint Falcon, mismo que se somete a una tarea de regulación basada en seguimiento, empleando polinomios de quinto orden para validar de forma experimental al control multiresolución, con la finalidad de realizar una comparación con otro tipo de control. Los resultados son comparados con un control PID discreto, mediante una medida de tendencia central y una medida de dispersión. Con ellos se busca ratificar y aprovechar las bondades que posee este tipo de control en sistemas robóticos, especialmente en un sistema háptico. Los resultados obtenidos permiten verificar y determinar dichos beneficios que el control multiresolución provee durante el seguimiento de trayectoria planificada, en comparación con controles lineales clásicos.
\end{abstract}

Palabras clave: control multiresolución, sistema háptico, control wavelet.

\section{Introducción}

En años recientes S. Parvez [1] desarrolló un nuevo controlador, el cual se basa en el análisis de la señal del error, mismo que es descompuesto en diferentes niveles de resolución, el cual nombró PID wavelet. El PID wavelet, nombrado así por tener ciertas similitudes con el control PID clásico (Proporcional-IntegralDerivativo), considera la señal de error (diferencia entre la señal de referencia y la señal de salida del sistema) para procesarla (obtener la derivada, la integral y una parte proporcional de la señal de error) y entregar una señal de control en función del error y una serie de ganancias previamente calculadas. Por otra parte el control PID wavelet no contempla solamente tres términos, sino que es posible obtener tantos como se requiera dependiendo del nivel de resolución al que se someta la señal de error. Por otra parte, los controles multiresolución (categoría en el que recaen no solo el PID wavelet sino una gran variedad de controles que utilizan técnicas de análisis multiresolución basada en la teoría wavelet, e.g. control wavelet auto-sintonizado con una wavenet [2], control PD-Wavelet+I [3], 
control P-wavelet+I [4], control de modos deslizantes+filtro wavelet [5], PID wavenet [6]) permiten un mejor análisis de la señal de error, al descomponer a diversos niveles de escala-frecuencia dicha señal. Es por ello que uno de los beneficios que han brindado estos controles es la inmunidad al ruido, lo que permite entregar una señal de control más suave que aquella entregada en otro tipo de control sin la necesidad de una etapa de filtrado y que a su vez garantiza prolongar la vida útil de los actuadores de la planta debido al libre chatering. Después de su aparición y gracias a estos beneficios ha sido implementado para el control de diversos sistemas como en [7], [8], [9], [10] y [11].

El artículo está seccionado de la siguiente manera: en la Sección 1 se tiene una breve introducción sobre los controladores multiresolución; en la Sección 2 se presenta la arquitectura del control multiresolución para un sistema SISO y para un sistema MIMO; mientras que en la Sección 3 se da a conocer al sistema háptico Novint Falcon donde se implementa el control; en la Sección 4 se da a conocer las estrategias de control empleadas, así como también la plataforma empleada y los resultados de las pruebas experimentales; en la Sección 5 se discuten los resultados y en la Sección 6 se dan las conclusiones obtenidas del trabajo de investigación; en la última Sección se dan las referencias empleadas en este trabajo de investigación.

\section{Control multiresolución}

En esencia, un control multiresolución toma la señal de error, mediante técnicas de análisis multiresolución, descompone esta señal en diversas señales en el espacio de escala-frecuencia, estas señales posteriormente son escaladas y sumadas para obtener la señal de control.

$$
u(k)=\sum_{i=1}^{N+1} K_{i} * e_{i}(k)
$$

donde $e_{i}$ es el i-ésimo componente de la señal de error a un nivel $i$ de resolución en el espacio escala-frecuencia, $K_{i}$ es la ganancia correspondiente al i-ésimo componente de la señal de error y $N$ es el nivel de resolución al que se somete a la señal de error $e(k)$. De acuerdo al nivel de resolución $N$ que se tenga siempre se tendrá $N+1$ componentes de la señal de error $e(k)$.

Actualmente, todos los controladores multiresolución emplean el análisis multiresolución basados en la teoría wavelet para su diseño y únicamente requiere la señal de error. Ésta técnica siempre dará un componente de la señal de error de alta escala - baja frecuencia, un componente de baja escala - alta frecuencia y $N-1$ componentes de mediana escala - mediana frecuencia.

Los componentes de la señal de error a un nivel $N$ de descomposición son obtenidos mediante la siguiente ecuación:

$$
e(k)=\sum_{j=-\infty}^{\infty} c_{N, j} \phi_{N, j}(k)+\sum_{i=1}^{N-1} \sum_{j=-\infty}^{\infty} d_{i, j} \psi_{i, j}(k)+\sum_{j=-\infty}^{\infty} d_{N, j} \psi_{N, j}(k),
$$


donde: el primer término del lado derecho de la ecuación representa al componente de alta escala - baja frecuencia $\left(e_{1}(k)\right)$, el segundo término a los componentes de mediana escala- mediana frecuencia $\left(e_{2}(k), \ldots, e_{N}(k)\right)$ y el último término al componente de baja escala - alta frecuencia $\left(e_{N+1}(k)\right)$; las funciones $\phi$ (función de escalamiento) y $\psi$ (función wavelet) son funciones base wavelet que permiten generar subespacios ortonormales $V$ y $W$ en $L_{2}(\mathbb{R}) ; c$ y $d$ son los coeficientes de aproximación y los coeficientes de detalle en cada i-ésimo nivel de descomposición, respectivamente, y son calculados por [12]:

$$
\begin{aligned}
& c_{i, j}=\left\langle e(k), \phi_{i, j}(k)\right\rangle=\sum_{j=-\infty}^{\infty} e(k) \overline{\phi_{i, j}(k)}, \\
& d_{i, j}=\left\langle e(k), \psi_{i, j}(k)\right\rangle=\sum_{j=-\infty}^{\infty} e(k) \overline{\psi_{i, j}(k)} .
\end{aligned}
$$

El control multiresolución para un sistema de múltiples entradas y múltiples salidas (MIMO) se representa de la siguiente manera. Sea $U(k) \in \mathbb{R}^{m}$ el vector de control que contiene las señales de control para el sistema MIMO, $\operatorname{Em}(k) \in \mathbb{R}^{m \times(N+1)}$ es la matriz que contiene los componentes de la señal del error correspondiente a cada salida, $K(k) \in \mathbb{R}^{(N+1) \times m}$ es la matriz que contiene las ganancias del controlador, $N$ es el nivel de descomposición y $m$ es el número de entradas y salidas del sistema MIMO. El vector de control se calcula como:

$$
U_{i}(k)=\sum_{j=0}^{N+1} E m_{i, j}(k) * K_{j, i}, \quad i=1,2, \ldots, m .
$$

En la Figura 1 se muestra el esquema básico de un sistema control multiresolución para sistemas MIMO, donde $Y_{\text {ref }}$ y $Y$ es la referencia y la salida del sistema, respectivamente; e y $E m$ es la señal de error y los componentes de la misma, respectivamente; $K$ son las ganancias del control que posteriormente son multiplicadas $E m$ para obtener la señal de control $U$; $P$ representa las perturbaciones externas que afectan al sistema y $R$ representa el ruido introducido a los sensores.

El control multiresolución posee estabilidad en lazo cerrado como se demostró en [13], sin embargo actualmente no existen métodos de sintonización. Una alternativa para sintonizarlo es empleando redes neuronales artificiales como en [4] y en [14].

\section{Dispositivo háptico Novint Falcon}

El dispositivo Novint Falcon [15] es del tipo paralelogramo, usa una variante de 3 grados de libertad $[x, y, z]$ de la configuración original del robot Delta, la cual es llamada traslacional, muy similar al modelo de Tsai [16]. Tiene incorporados efectores finales que pueden removerse fácilmente cuando el dispositivo está en 


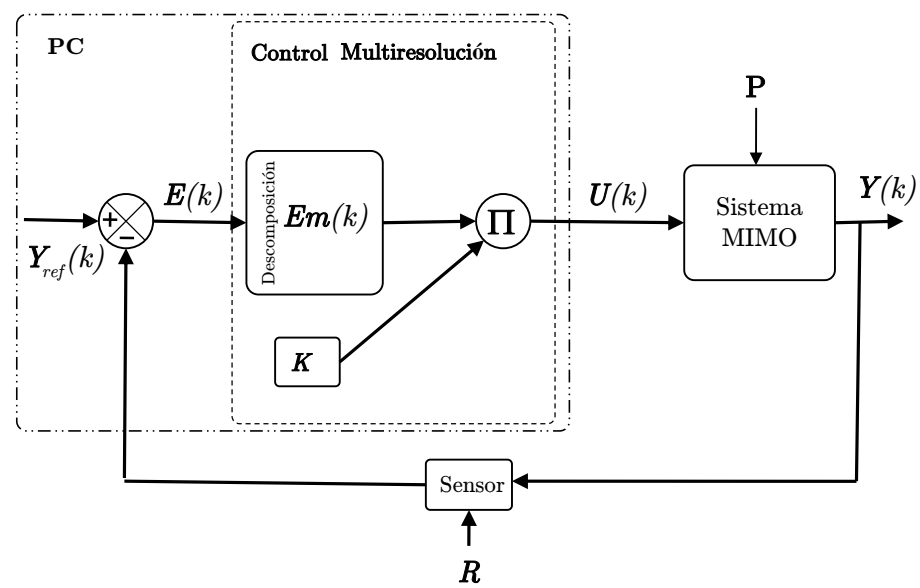

Fig. 1: Esquema básico de un sistema de control multiresolución para un sistema MIMO

uso y puede seguir trabajando de manera normal. Ejemplos de estos efectores son: una pistola y un sujetador esférico (Ver Figura 2). El primero se usa constantemente en videojuegos y el segundo sirve más como apuntador.

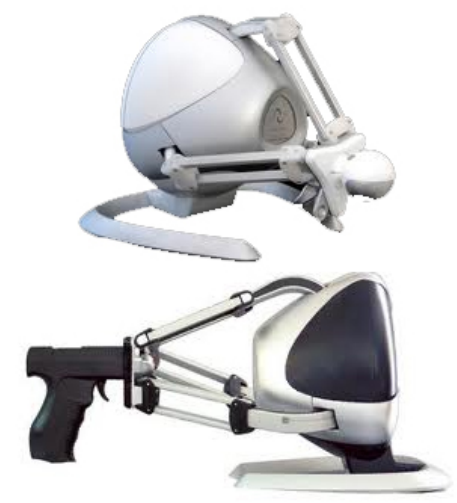

Fig. 2: Efectores del dispositivo Novint Falcon.

El dispositivo cuenta con las siguientes características: 
- El área de trabajo es aproximadamente de $10.16 \mathrm{~cm}$ x $10.16 \mathrm{~cm}$ x $10.16 \mathrm{~cm}$ (Ver Figura 3).

- Las capacidades de fuerza son mayores a 0.90 kilogramos.

- Suministra 400 dpi (puntos por pulgada) en resolución de posición cartesiana.

- Interfaz de comunicación USB 2.0.

- 1.81 kilogramos de peso.

- Consume 30 watts, $110 \mathrm{v}-240 \mathrm{v}, 50 \mathrm{~Hz}-60 \mathrm{~Hz}$.

- Usa 3 motores Mabuchi RS-555PH-15 con codificadores ópticos de 320 líneas por revolución.

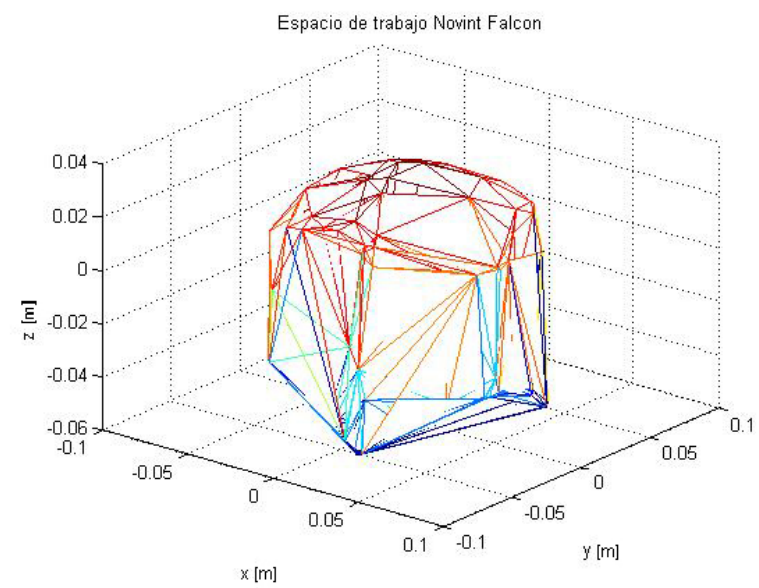

Fig. 3: Área de espacio de trabajo mesurada tomando puntos aleatorios.

El dispositivo se comunica con la computadora a través de la interfaz USB 2.0, la computadora envía y recibe señales de control las cuales son interpretadas por el sistema de control del dispositivo y enviado a los motores. Estos, a su vez, cuentan con codificadores ópticos para la lectura de la posición angular. El desarrollo de la aplicación se realiza bajo el lenguaje $\mathrm{C}++$ con ayuda del API en su versión 2.1.3 del HDAL, el cual otorga al usuario programador dos visiones, una a bajo nivel y otra a alto nivel para su uso.

La HDAL (Capa de abstracción del dispositivo háptico) es el modelo de arquitectura con el que está implementado el dispositivo Novint Falcon el cual consiste en un sistema de capas que se comunican con el software de aplicación.

La aplicación está conformada de dos principales componentes: la simulación gráfica compuesta de ambientes virtuales y el componente de simulación háptica que contiene la lógica de control del dispositivo. La aplicación se comunica con la HDAL a través de su API, a la cual se le envía como parámetro una función que es llamada a cada lectura de los servos (actuadores), con lo que se proporciona la posición y la fuerza del dispositivo en tiempo real (Figura 4). 


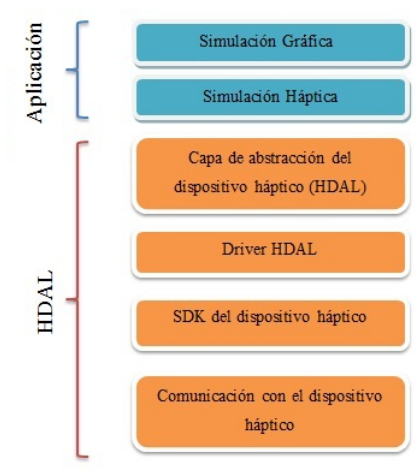

Fig. 4: Estructura de la HDAL del dispositivo Novint Falcon.

\section{Resultados experimentales}

Los resultados experimentales se basan en la aplicación de dos estrategias de control al dispositivo háptico Novint Falcon. Para ello se generan consignas de movimiento basadas en la planificación de una tarea que consta del seguimiento de dos trayectorias.

T1: una trayectoria desde el punto inicial $P_{0}$ hasta el punto $P_{1}$, es una trayectoria de referencia $y_{r e f}$ generada por el polinomio $\xi$, como

$$
\xi(k)=10\left(k / k_{b}\right)^{3}-15\left(k / k_{b}\right)^{4}+6\left(k / k_{b}\right)^{5},
$$

donde $k_{b}$ es el tiempo de convergencia, que para este caso es de 1 segundo, por lo tanto la trayectoria de referencia es

$$
Y_{\text {ref }}(k)=P_{0}+\left(P_{1}-P_{0}\right) * \xi(k),
$$

T2: consta de una circunferencia como trayectoria de referencia $Y_{\text {ref }}$ exclusivamente sobre el plano X-Y, iniciada en el punto $P_{1}$ y terminada sobre el mismo punto, las ecuaciones paramétricas que la definen son:

$$
\begin{aligned}
& Y_{x_{\text {ref }}}(k)=r \cos \left(2 \pi\left(k-k_{b}\right) / T\right) \\
& Y_{y_{\text {ref }}}(k)=r \sin \left(2 \pi\left(k-k_{b}\right) / T\right) \\
& Y_{z_{\text {ref }}}(k)=0,
\end{aligned}
$$

donde $T$ es el tiempo de duración de la trayectoria y $r$ es el radio de la circunferencia, que para este caso es $T=10$ segundos y $r=2 \mathrm{~cm}$.

\subsection{Estrategias de control}

Las estrategias de control aplicadas al dispositivo háptico son: el control multiresolución y el control PID discreto. Para el control multiresolución se utiliza la ecuación (5). De acuerdo a la realización de una serie de pruebas empleando 
diferentes niveles de descomposición $(N)$, se determinó utilizar $N=5$, ya que se obtiene una mejor resolución y una señal de control suave, suprimiendo la señal de ruido. Además se utilizan los coeficientes de filtro Daubechies de soporte compacto de orden 2 dados en [17]:

Tabla 1: Coeficientes del filtro Daubechies de orden 2

\begin{tabular}{|l|l|}
\hline $\mathrm{n}$ & Coeficientes del filtro pasa bajas \\
\hline 0 & 0.48296291 \\
1 & 0.83651630 \\
2 & 0.22414386 \\
3 & -0.12940952 \\
\hline
\end{tabular}

El PID discreto se rige por la siguiente ecuación [18]:

$$
\begin{aligned}
U_{i}^{P I D}(k)= & U_{i}^{P I D}(k-1)+K_{P, i}^{P I D}[e(k)-e(k-1)]+K_{I, i}^{P I D} e(k)+ \\
& K_{D, i}^{P I D}[e(k)-2 e(k-1)+e(k-2)],
\end{aligned}
$$

donde $U_{i}^{P I D}(k) \in \mathbb{R}$ es el i-ésima señal de control del PID, $K_{P, i} \in \mathbb{R}$ es la i-ésima ganancia para la parte proporcional, $K_{I, i} \in \mathbb{R}$ es la i-ésima ganancia para la parte integral y $K_{D, i} \in \mathbb{R}$ es la i-ésima ganancia para la parte derivativa.

Las ganancias de ambas leyes de control son obtenidas de manera experimental para el sistema no lineal Novint Falcon y son dadas en el Cuadro 2 y 3 .

Tabla 2: Valores de las ganancias del control multiresolución.

\begin{tabular}{|c|c|c|c|c|c|c|}
\hline Ganancias del control multiresolución & $K_{1}$ & $K_{2}$ & $K_{3}$ & $K_{4}$ & $K_{5}$ & $K_{6}$ \\
\hline Ganancias para $U_{1}$ & 30 & 10 & 0 & 0 & 0 & 0 \\
\hline Ganancias para $U_{2}$ & 30 & 10 & 0 & 0 & 0 & 0 \\
\hline Ganancias para $U_{3}$ & 30 & 10 & 0 & 0 & 0 & 0 \\
\hline
\end{tabular}

Tabla 3: Valores de las ganancias para el PID discreto.

\begin{tabular}{|c|c|c|c|}
\hline Ganancias del PID discreto & $K_{P, i}$ & $K_{I, i}$ & $K_{D, i}$ \\
\hline Ganancias para $U_{1}$ & 12.5 & 0 & 4.5 \\
\hline Ganancias para $U_{2}$ & 12.5 & 0 & 4.5 \\
\hline Ganancias para $U_{3}$ & 12.5 & 0 & 4.5 \\
\hline
\end{tabular}




\subsection{Plataforma experimental}

La plataforma experimental está constituida por dos elementos importantes: uno corresponde al dispositivo Novint Falcon y el segundo al de un equipo de cómputo que tiene las siguientes características.

- Procesador AMD Turion x2 a $2.00 \mathrm{GHz}$.

- $2.00 \mathrm{~Gb}$ en RAM DDR II 667 mhz.

- Sistema operativo windows 7 service pack 1.

- Tarjeta de vídeo ATI Radeon x1250 con 128 MB de memoria compartida.

- Controlador ATI SB600-OCHI USB 2.0.

\subsection{Resultados}

En los cuadros 4 y 5 , se dan a conocer los resultados obtenidos que corresponden a la media $(\bar{\mu})$ y la desviación estándar $(\sigma)$ de la señal de error en los tres ejes para cada uno de los controles, respectivamente.

Tabla 4: Media de la señal de error

\begin{tabular}{|c|c|c|c|}
\hline Eje & Control Multiresolución & PID discreto & unidad \\
\hline $\mathrm{x}$ & 0.0027 & 0.0029 & $\mathrm{~cm}$. \\
$\mathrm{y}$ & 0.0074 & 0.0121 & $\mathrm{~cm}$. \\
$\mathrm{z}$ & 0.0425 & 0.0494 & $\mathrm{~cm}$. \\
\hline
\end{tabular}

Tabla 5: Desviación estándar de la señal de error

\begin{tabular}{|c|c|c|c|}
\hline Eje & Control Multiresolución & PID discreto & unidad \\
\hline $\mathrm{x}$ & 0.0043 & 0.0047 & $\mathrm{~cm}$. \\
$\mathrm{y}$ & 0.0030 & 0.0041 & $\mathrm{~cm}$. \\
$\mathrm{z}$ & 0.0059 & 0.0073 & $\mathrm{~cm}$. \\
\hline
\end{tabular}

En la Figura 5 se presenta el desempeño del efector final del dispositivo háptico Novint Falcon durante el seguimiento de la tarea T1-T2 empleando el control multiresolución. En la Figura 6 es posible observar el comportamiento del error de posición y sus componentes obtenidas mediante la técnica de multiresolución, donde el componente principal de mayor escala pero de baja frecuencia $\left(e_{x 1}\right)$, es representado por el primer término de la ecuación 2 , y los componentes de mediana escala y mediana frecuencia $\left(e_{x 2}, \ldots, e_{x 5}\right)$, son representado por el segundo término de la ecuación 2 y al término de menor escala y mayor frecuencia 
$\left(e_{x 6}\right)$, es representado por el tercer término de la ecuación 2. Estos términos son escalados para calcular la señal de control $U_{1}$.

Además se realiza un acercamiento en el intervalo [1.8, 1.9] con la finalidad de percibir con mayor claridad los componentes del error.

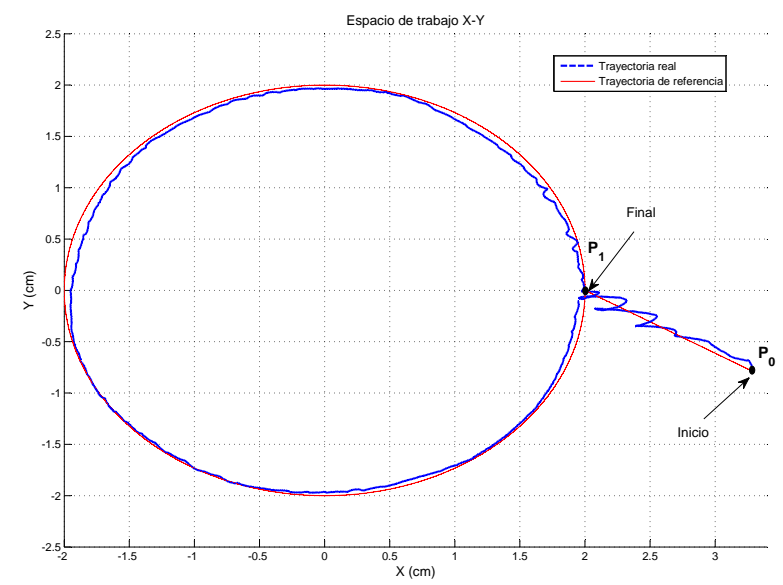

Fig. 5: Trayectoria del sistema háptico en el espacio de trabajo

En la Figura 7 se muestra la señal de control generada por el control multiresolución $U_{1}$ y sobre la misma figura se realiza una ampliación en el intervalo de tiempo de [2, 3.2], donde se observa cambios suaves de la señal de control. Finalmente en la Figura 8, se observa la señal de control PID discreto $U_{1}^{P I D}$ y sobre la misma se realiza una ampliación en el intervalo de tiempo de $[2,3.2]$, en el cual se aprecia la presencia de una señal que oscila a una alta frecuencia en comparación con el control multiresolución.

\section{Discusión}

A partir de los datos obtenidos de la media de la señal de error y su dispersión en los tres ejes de ambos controles, el sistema presenta robustez y estabilidad en cada instante de tiempo manteniendo en algunas ocasiones un error nulo para el control multiresolución, como se observa de manera gráfica en la Figura 5, donde el efector final del dispositivo Novint Falcon trata de seguir de manera suave, evitando cambios repentinos en la ejecución de la tarea T1-T2. Sin embargo se aprecian pequeñas y constantes oscilaciones durante la mayor parte del seguimiento, esto es provocado por las dinámicas (gravedad, tribología, Coriolis, 


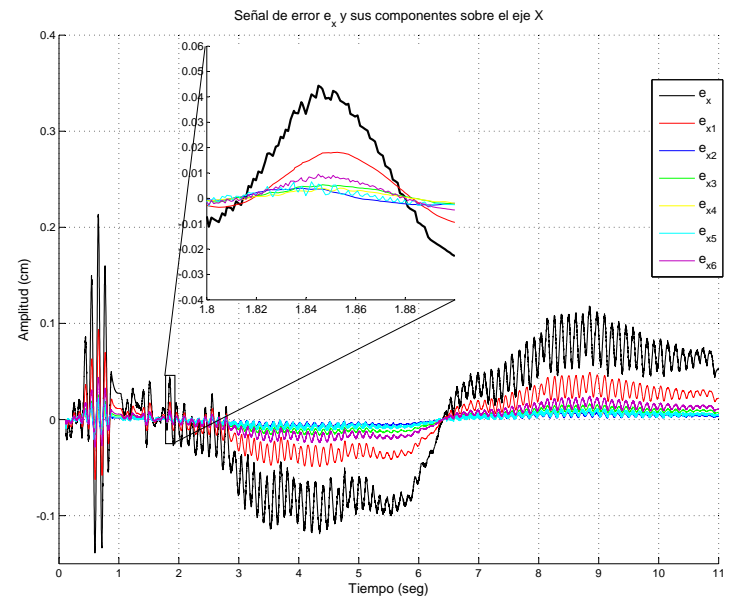

Fig. 6: Señal de error $e_{x}$ y sus componentes a un nivel de descomposición de $\mathrm{N}=5$

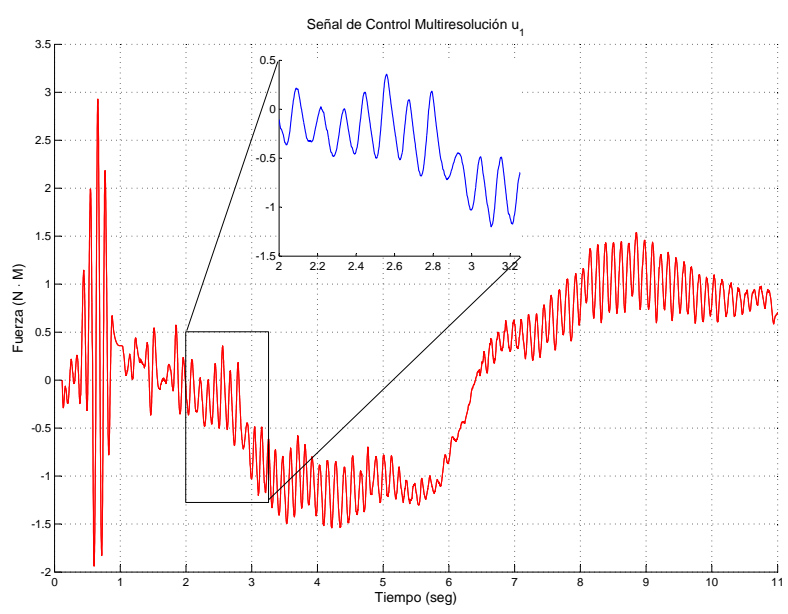

Fig. 7: Señal de control $U_{1}$ generada por el control multiresolución

y centrípetas) del dispositivo, mismas que no logran ser compensadas por el control, el cual maneja ganancias constantes durante toda la tarea.

La Figura 6 nos ayuda a seleccionar de alguna manera las ganancias del control multiresolución, ya que los términos que contiene altas frecuencias pueden ser escaladas con ganancias muy pequeñas y en algunos casos nulas, para evitar introducir el ruido al dispositivo háptico por medio de la señal de control, por 


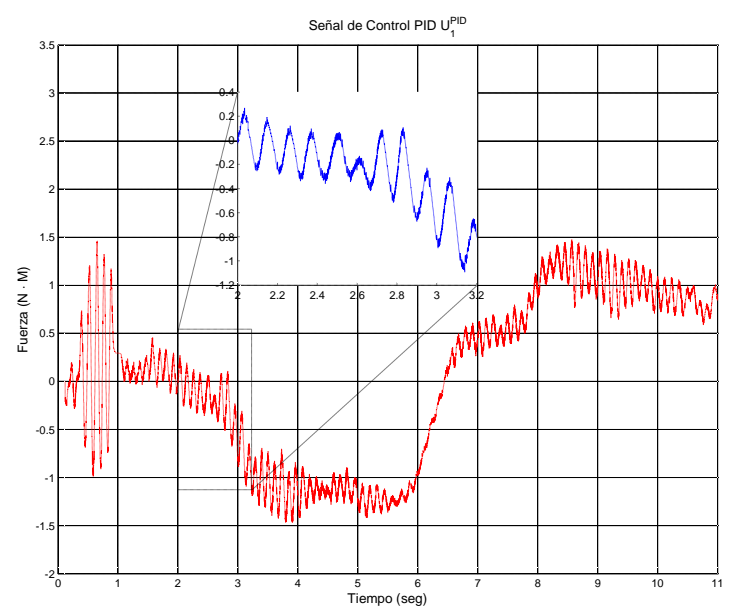

Fig. 8: Señal de control $U_{1}^{P I D}$ generada por el control PID discreto

tal razón las ganancias $K_{3}, K_{4}, K_{5}$ y $K_{6}$ en las tres señales de control dadas en el Cuadro 2 son cero. Por lo tanto, se obtiene una señal de control suave debido a proceso de selección de las ganancias de anular los componentes de la señal de error de alta frecuencias, eliminando señales no deseadas (chatering) que pueden causar efectos en los mecanismos del sistema. Mientras que en la Figura 8 se muestra como la señal de control del PID contiene todavía ruido proveniente de la señal de error y amplificado por la parte derivativa del mismo.

\section{Conclusiones}

El control multiresolución presenta ventajas sobre la gran mayoría de los controles clásicos, ya que su comportamiento tiene la característica de ser suave (sin chatering). Ésto se debe a que muestra inmunidad al ruido, mismo que es introducido por los sensores ópticos del dispositivo, actuadores y la estructura dinámica del dispositivo. También, porque a diferencia de la mayoría de los sistemas de control emplean una etapa de filtrado para cancelar los componentes de alta frecuencia de la señal de error, mientras que en el control multiresolución esta etapa no es requerida. Así, esas señales no son eliminadas, sino estudiadas de mejor manera para elegir cuáles pueden ser incorporadas y cuáles no al sistema, de esta forma no se pierde información. Además, en el control multiresolución no se requiere del conocimiento de las velocidades lineales o articulares para el diseño del mismo, lo que minimiza el cálculo matemático en el dispositivo, mismo que requiere un procesamiento adicional. Sin embargo, se carecen de técnicas o procedimientos para el cálculo ideal de sus ganancias. Una opción sería el uso de redes neuronales, que permitan auto-sintonizar las ganancias en toda la 
ejecución de la tarea y sobre todo el espacio de trabajo del dispositivo háptico. En consecuencia un trabajo futuro es la implementación de redes neuronales que permitan sintonizar las ganancias del control multiresolución en tiempo real, realizando además pruebas experimentales ante perturbaciones externas.

\section{Referencias}

1. Parvez, S., Gao, Z.: A Wavelet-Based Multiresolution PID Controller. IEEE Transactions on Industry Applications. 41(2), 537-543 (2005)

2. Cruz, J. A., Ramos, L. R., Espejel, M. A.: A Self-Tuning of a Wavelet PID Controller. pp. 73-78, IEEE Press, Puebla, México (2010)

3. Parvez, S.: Advanced Control Techniques for Motion Control Problem. Cleveland State University [Ph. D. Dissertation], Cleveland, Ohio, USA (2003)

4. Cruz, J. A.: Diseño y Aplicaciones de un Control PID Wavelet. Universidad Autónoma del Estado de Hidalgo [Tesis de Grado de Maestría], Pachuca, Hidalgo, México (2009)

5. Jarillo, A., Domínguez, O. A., Cruz, J. A., Ramos, L. E., Mijangos, T. S.: Design and Experimental Result of a Wavelet Filter for High Performance in Trajectory of Robotc System. International Journal of Scientific and Engineering Research, 3(10), 1300-1306 (2012)

6. Sedighizadeh, M., Rezazadeh, A.: Adaptive PID Control of Wind Energy Conversion Systems Using RASPl Mother Wavelet Basis Function Networks. In: Proceedings of World Academy of Science, Engineering and Technology, 27, pp. 269-273 (2008)

7. Khan, M. A., Azizur Rahman, M.: Real-Time Implementation of a Wavelet Based Speed Controller for Induction Motor Drives. In: Canadian Conference on Electrical and Computer Engineering, pp. 546-549, IEEE Press, Vancouver, Canada (2007)

8. Khan, M. A., Hinchey, M. J., Azizur Rahman, M.: Implementation of Wavelet Controller for Battery Storage System of Hybrid Electric Vehicle. In: IEEE Industry Applications Society Annual Meeting, pp. 1-8, IEEE Press, Houston, Texas (2009)

9. Cruz, J. A., Jarillo, A., Ramos, L. E.: Control de un Robot Manipulador Usando Control Wavelet: Experimental. In: VI Semana Nacional de Ingeniería, pp. 33-42, Oaxaca, México (2010)

10. Jeevan, L. J., Malik, V.: A Wavelet Based Multi-Resolution Controller. In: Journal of Emerging Trends in Computing and Information Sciences, pp. 17-21 (2011)

11. Yao, C. Zhang, G.: A Multi-Resolution PID Controller based on the wavelet transform. In: Advanced Materials Research, pp. 632-636 (2012)

12. Goswami, J. C., Chan, A. K.: Fundamentals of Wavelet: Theory, Algorithms and Aplications. John Wiley and Sons, New Jersy, USA (2010)

13. Roldan, H., Itzá, B. A., Ramos, L. E.: Análisis de estabilidad de un control PID wavelet. Revista Internacional de sistemas Computacionales y Electrónicos, 3(6), 27-32, Hidalgo, México (2011)

14. Khan, M. A., Azizur Rahman, M. A.: A Novel Neuro-Wavelet Based Self-Tuned Wavelet Controller for IPM Motor Drives. In: IEEE Industry Applications Society Annual Meeting, 2008, pp. 1-8. IEEE Press, Edmonton, Canada (2008)

15. Novint Technologies, Inc.: http://www.novint.com

16. Tsai, L. W.: A Parallel Manipulator with Only Translational Degrees of Freedom. In: ASME Design Engineering Technical Conferences (1996)

17. Daubechies, I.: Ten Lectures on Wavelets. SIAM, Philadelphia, USA (1992)

18. Visioli, A.: Practical PID Control. Springer-London, England (2006) 